\title{
Rice annexin OsANN4 interacting with OsCDPK24, reduces root aerenchyma formation by modulating $\mathrm{H} 2 \mathrm{O} 2$ under $\mathrm{ABA}$
}

Qian Zhang ${ }^{1}$, Tao Song ${ }^{1}$, Can Guan ${ }^{1}$, Yingjie Gao ${ }^{1}$, Jianchao $\mathrm{Ma}^{1}$, Zhiguang $\mathrm{Qi}^{1}$, Jingze Liu ${ }^{1}$, Ying $\mathrm{Zhu}^{2}$, Zhengge Zhu ${ }^{1 *}$

1 Hebei Key Laboratory of Molecular and Cellular Biology, Key Laboratory of Molecular and Cellular Biology of the Ministry of Education, College of Life Science, Hebei Normal University, Hebei Collaboration Innovation Center for Cell Signaling, Shijiazhuang, Hebei, 050024, China 2 The Institute of Viral and Biotechnology, Zhejiang Academy of Agricultural Sciences, Hangzhou, 310021, China

Corresponding author: Zhengge Zhu, E-mail: zhuzhengge@ hebtu.edu.cn, Tel: 0311-8078 7528;

Qian Zhang: xjzz2005.wan@163.com; Tao Song: hebtusongtao@126.com; Can Guan: thisisguancan@163.com; Yingjie Gao: gyj@hebtu.edu.cn; Jianchao Ma: 1030276036@qq.com; Zhiguang Qi: zhiguangqi@126.com; Jingze Liu: liujingze@hebtu.edu.cn; Ying Zhu: yzhuzaas@163.com.

Date of submission: 2019/1/12

Number of figures: 7

Word count: 5528

Supplementary figures: 4

Supplementary table: 1 
Highlight OsANN4 enhances SOD and CAT activities to scavenge $\mathrm{H}_{2} \mathrm{O}_{2}$ to alleviate the formation of aerenchyma under ABA treatment. OsCDPK24 interacts with and phosphorylates OsANN4, this interaction strengthened under ABA treatment.

\begin{abstract}
Plant annexins are calcium- and lipid-binding proteins that have multiple functions, and a significant amount of research on plant annexins has been reported in recent years. However, the functions of annexins in diverse biological processes in rice are largely unclear. Herein, we report that $O S A N N 4$, a calcium-binding rice annexin protein, is a substrate for OsCDPK24, and the OsANN4 phosphorylation site is the 13th serine, which is a key site for phosphorylation. Most strikingly, abscisic acid (ABA) promotes the interaction between OsANN4 and OsCDPK24. Moreover, knocking down OsANN4 by RNA interference resulted in visible and invisible phenotypes with exogenous ABA treatment, such as shorter shoots of seedlings, less lateral roots, earlier root aerenchyma formation and so on. The further analyzed results showed that decreased superoxide dismutase (SOD) and catalase (CAT) activity of the RNAi lines, which control $\mathrm{H}_{2} \mathrm{O}_{2}$ accumulation for redox homeostasis, and further promoted earlier aerenchyma formation of the root. These results suggest that a proposed molecular mechanism exists between OsANN4 and $\mathrm{H}_{2} \mathrm{O}_{2}$ production to response $\mathrm{ABA}$.
\end{abstract}

Keywords : Annexin, Calcium-dependent protein kinase, Phosphorylation, $\mathrm{H}_{2} \mathrm{O}_{2}$, Abscisic acid, Oryza sativa

\title{
Introduction
}

Abscisic acid (ABA), a well-known long-distance signaling molecule utilized for communication between plant roots and shoots under water-deficient conditions, is also considered a hormone that plays a critical role in abiotic stress tolerance in plants (Pekic et al., 1995; Cutler et al., 2010). Recently, many mediators involved in ABA signaling, such as ABA receptors (McCourt and Creelman, 2008; Klingler et al., 
2010; He et al., 2014) and targets of ABA receptors (Bueso et al., 2014; Ye et al., 2017), have been characterized. Since the identification of the steroidogenic regulatory protein (StAR)-related lipid-transfer (START) domain as a candidate ABA receptor (Ma et al., 2009; Santiago et al., 2009; Nishimura et al., 2010), pyrabactin resistance 1 (PYR1) and PYR1-like 1-13 (PYL1-PYL13) have been considered as key components of the core ABA signaling pathway (Fujii et al., 2009; Ma et al., 2009; Park et al., 2009; Zhao et al., 2013). ABA receptors may inhibit the activity of phosphatase 2Cs (PP2Cs) and result in the activation of Suc nonfermenting-1-related protein kinase 2 (SnRK2s), further promoting the expression of some downstream transcription factors or membrane ion channels (Melcher et al., 2010; Ng et al., 2014).

Exposure to abiotic stresses, such as salt, drought and oxidative stress, adversely affects plant growth and crop productivity. ABA functions as an important phytohormone that has long been known to play a critical role in stress responses (Giraudat et al., 1994; Himmelbach et al., 2003) to regulate the expression of many genes, leading to complex physiological and metabolic responses that enable plants to confer tolerance to abiotic stress (Cutler et al., 2010; Umezawa et al., 2010). Increasing evidence shows that ABA-enhanced abiotic stress tolerance might be associated with the induction of antioxidant defense systems, including reactive oxygen species (ROS) scavenging enzymes, such as catalase (CAT), superoxide dismutase (SOD), ascorbate peroxidase (APX) and non-enzymatic antioxidants (Jiang and Zhang, 2002; Miao et al., 2006; Neill et al., 2008; Xing et al., 2008; Miller et al., 2010; Ozfidan et al., 2012; Ding et al., 2013). Especially the recent study shows in the presence of $\mathrm{ABA}$, the increased production of $\mathrm{H}_{2} \mathrm{O}_{2}$ activates a calcium-dependent protein kinase ( $\mathrm{Ni}$ et al., 2018), it suggests $\mathrm{ABA}$ induced $\mathrm{H}_{2} \mathrm{O}_{2}$ production to play a key role in response to abiotic stress.

Annexins belong to an evolutionarily conserved multi-gene protein superfamily comprising $\mathrm{Ca}^{2+}$-dependent phospholipid-binding proteins. Calcium ions bind to annexin molecules mainly via coordination sites termed type II ("AB") high-affinity $\mathrm{Ca}^{2+}$-binding sites. In addition, annexins may contain two other binding sites with lower affinity, named the type III "B" and "DE" sites (Moss and Morgan, 2004). 


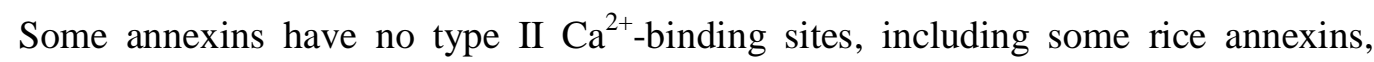
indicating that different protein conformations and different mechanisms for binding phospholipids may exist.

Plant annexins are reportedly tissue-specific, and their expression is regulated developmentally. Recent results also suggested that annexins play an important role in plant stress responses (Clark et al., 1998; Lee et al., 2004; Clark et al., 2012; Jami et al., 2012; Richards et al., 2014; Qiao et al., 2015), and several plant annexins have been demonstrated to play specific roles in ABA treatment and osmotic stress. The alfalfa (Medidago sativa) annexin gene AnnMs 2 was first reported to be activated by drought (Kovacs et al., 1998). AtANN1 and AtANN4 play important roles in osmotic stress and ABA signaling in a $\mathrm{Ca}^{2+}$-dependent manner (Jami et al., 2012). AtANN1 was proven to act as an $\mathrm{H}_{2} \mathrm{O}_{2}$ sensor (Laohavisit et al., 2010). The expression of annexin upon ROS regulation have been identified in several organism types, including plants and animals (Richards et al., 2014). The rice OsANN1 gene shows the ability to confer heat and drought stress tolerance by modulating antioxidant accumulation under abiotic stresses (Qiao et al., 2015).

\section{Sequence analysis revealed that annexin may have a post-transcriptional} modification site such as a phosphorylation site, which may be a substrate for protein kinases (Jami et al., 2012). Evidence obtained using a tandem affinity purification approach to identify protein complexes suggests that annexin may interact with various kinases, including receptor-like kinase, sterile-20 (Ste20)-like kinase, calcium/calmodulin-dependent protein kinase and casein kinase (Rohila et al., 2006). In addition to the MAPK cascade, $\mathrm{Ca}^{2+}$ signaling is another critical pathway triggered by environmental stimuli and developmental cues. Calcium-dependent protein kinases (CDPKs) are serine/threonine protein kinases that function as one of the best characterized $\mathrm{Ca}^{2+}$ sensors in plants. Previous studies suggested that CDPKs are involved in the responses of plants to various abiotic stresses, including salt, drought, hormonal stimuli and oxidative stress (Kobayashi et al., 2007; Zhu et al., 2007; Asano et al., 2012; Rahoui et al., 2017). OsCPK24 (same as OsCDPK24), the rice CDPK gene, increases the tolerance of rice seedlings by reducing the thioltransferase activity 
of OsGrx10 to increase proline and glutathione contents under cold stress (Liu et al., 2018). The transcription factor protein OsDi19-4 interacts with OsCDPK14/OsCPK24 and is further phosphorylated by OsCDPK14/OsCPK24, acting in response to ABA signaling (Wang et al., 2016). However, the interactions between protein kinases CDPKs and annexins that are involved in the regulation of antioxidant defenses in ABA signaling remain to be determined.

The aerenchyma is a normal morphological structure utilized by plants to adapt to the waterlogging and submergence of soil, promoting internal oxygen diffusion (Colmer, 2003; Licausi et al., 2011; Licausi, 2013). Ultra structural results suggest that cortical cell death occurs constitutively (Joshi and Kumar, 2012) via a process termed constitutive aerenchyma formation. Under oxygen-deficient conditions, many plant roots will induce aerenchyma development to ensure internal oxygen transport from the shoots to roots, thus facilitating gas diffusion (Campbell and Drew, 1983; Colmer, 2003). Aerenchyma formation is regulated through reactive oxygen species (ROS) in plants (Colmer and Pedersen, 2008; Rajhi et al., 2011; Yamauchi et al., 2014). Hydrogen peroxide $\left(\mathrm{H}_{2} \mathrm{O}_{2}\right)$, a type of ROS, plays a key role in programmed cell death and lysigenous aerenchyma formation (Yamauchi et al., 2017).

In this study, we identified a putative annexin family gene in rice, OsANN4. We investigated that OsANN4 alleviates ABA-induced root aerenchyma formation via modulating $\mathrm{H}_{2} \mathrm{O}_{2}$ accumulation. Moreover, OsANN4, a calcium-binding annexin protein, is an interacting protein of kinase OsCDPK24 and further be phosphorylated by OsCDPK24, and responses to ABA treatment. Thus, we have discovered a proposed mechanism for annexin and protein kinase in $\mathrm{H}_{2} \mathrm{O}_{2}$ production in aerenchyma formation to response ABA.

\section{Materials and methods}

\section{Vector construction for recombinant protein expression and rice transformation}

Total RNA was extracted from 7-day-old seedlings of WT plants. Full-length OsANN4 cDNA without the stop codon was amplified with primers P1 and P2 via 
reverse transcription PCR. For sequencing and subcloning, the products were initially ligated into a PMD18-T vector. After sequencing, the fragment was cut with $\mathrm{Xba \textrm {I } \text { and }}$ KpnI and inserted into the PMDC83 expression vector driven by a CaMV35S promoter. To construct the RNA interference vector, the 347-bp coding sequence of OsANN4 was amplified with primers $\mathrm{P} 3$ and P4, digested with SacI and SpeI followed by BamHI and KpnI, and subsequently ligated into the pTCK303 vector.

For the $\mathrm{Ca}^{2+}$-binding assay, the expression vector pET28a-OsANN4 was constructed. Primers P5 and P6 were designed, and the full-length cDNA encoding OsANN4 was subcloned into E. coli between the EcoRI and HindIII sites in an orientation such that the cDNA sequence was followed by a His tag.

For the pull-down assay, the expression vector pGEX4T-1-OsANN4 was constructed. The full-length cDNA encoding OsANN4 was ligated into pGEX4T-1 using primers P5 and P6 to obtain the cDNA sequence followed by a GST tag. The expression vector pET28a-OsCDPK24 was constructed by using primers P7 and P8.

For the LCI assay, the full-length cDNA encoding OsANN4 was ligated into pCAMBIA-Cluc by using primers $\mathrm{P} 9$ and P10, and OsCDPK24 was ligated into pCAMBIA-Nluc by using primers P11 and P12.

For the construction of pET28a-OsANN4(S13A), the primers P5 and P6 were used and the vector of OsANN4(S13A)-Cluc was constructed by using primers P9 and P10. Site-directed mutagenesis was finished by a fast mutagenesis kit (Tiangen, China).

All primer sequences used in these experiments are listed in Table S1. The expression vectors pET28a-OsANN4 and pGEX4T-1-OsANN4 were then transformed into the E. coli Rosetta strain. In addition, other constructs were introduced into Agrobacterium EHA105 and transformed into rice calli. Transgenic rice plants were generated as previously described.

\section{Plant materials and stress treatment}

The rice (Oryza sativa subsp. japonica) cultivar Nipponbare was used in this study and as the WT control in all experiments. Rice seeds were surface-sterilized with 50\% 
$177 \mathrm{NaClO}$ for $20 \mathrm{~min}$, rinsed 10 times with sterile distilled water and then grown on $1 / 2$

178 MS medium. The rice plants were grown in a standard culture solution in a

179 greenhouse with a light/dark cycle of $16 / 8 \mathrm{~h}$ and $50 \%$ relative humidity at $28 / 25^{\circ} \mathrm{C}$

180 (day/night). For the ABA sensitivity assay, the seeds were planted on 1/2 MS medium

181 supplemented with $10 \mu \mathrm{M}$ ABA for 12 days. Control seeds were planted on $1 / 2 \mathrm{MS}$

182 medium and cultured with water after 12 days.

RNA isolation, RT-PCR, and quantitative RT-PCR analysis

185 Total RNA from different tissues of rice plants was isolated using the TaKaRa RNAiso Plus kit. Purified RNA (2 $\mu \mathrm{g}$ ) was incubated with DNase 1 ( RNase-Free DNase, Thermo Fisher, USA) at $37^{\circ} \mathrm{C}$ for 30 min. First-strand cDNA was synthesized using the PrimeScript ${ }^{\mathrm{TM}}$ First Strand cDNA Synthesis Kit (TaKaRa, Japan) to perform RT-PCR. For qRT-PCR, $1 \mu \mathrm{g}$ purified total RNA was used to obtain the first-strand cDNA with the PrimeScript ${ }^{\mathrm{TM}}$ RT reagent Kit with gDNA Eraser (TaKaRa, Japan).

The Takara SYBR ${ }^{\circledR}$ Premix Ex Taq ${ }^{\mathrm{TM}}$ II was used to perform the qRT-PCR assay using primers P13 and P14. The C1000 Real-Time PCR instrument (Bio-Rad, USA) was used for qRT-PCR. OsACTIN1 (Os03g0718100) was used as an internal control for the normalization of all data by using Primers P15 and P16 in this experiment. Three independent biological replicates were assayed.

\section{Sectioning and microscopy of root tips}

Adventitious roots were obtained from 1/2 MS medium supplemented with $10 \mu \mathrm{M}$ ABA. After embedding in resin (SPI, USA), the embedded materials were measured and marked at $3 \mathrm{~mm}$ and $5 \mathrm{~mm}$ from the root tip. Then the transverse sections of $1 \mu \mathrm{m}$ thickness were acquired on a RM 2265 rotary microtome (Leica, Germany). And the resin section was stained with toluidine blue, and examined and photoed by a DVM6a 3D microscope (Leica, Germany). 
$\mathrm{H}_{2} \mathrm{O}_{2}$ content in rice seedlings was determined using the Hydrogen Peroxide Assay

Kit (Beyotime, China). According to the instructions, the samples were fully homogenized in lysis buffer. Fifty microliters of supernatant was added to $100 \mu \mathrm{l}$ of test solution and then placed at $30 \square$ for 30 min. Readings at a wavelength of $560 \mathrm{~nm}$ were measured immediately. Then, $\mathrm{H}_{2} \mathrm{O}_{2}$ content was calculated according to the standard curve.

To determine the total soluble protein content, rice seedlings $(0.2 \mathrm{~g}$, fresh weight, FW) were homogenized in $1.0 \mathrm{ml}$ of extraction buffer $(50 \mathrm{mM}$ phosphate, $1 \%(\mathrm{w} / \mathrm{v})$ polyvinyl pyrrolidone, $\mathrm{pH}$ 7.8) in a mortar on ice. The plant tissue was ground into a homogenate and then centrifuged at $4{ }^{\circ} \mathrm{C}$ for $20 \mathrm{~min}$ at $8000 \mathrm{rpm}$. The supernatants were used for assaying the protein content and enzyme activities. The total soluble protein contents were then determined by the Bradford method using bovine serum albumin (BSA, Solarbio, China) as the standard. All spectrophotometric analyses were performed on a METASH UV-5200PC spectrophotometer (Shanghai, China).

DAB staining was performed following a published method with some modifications. 7-day-old rice seedlings were treated with $10 \mu \mathrm{M} \mathrm{ABA}$, and then the leaves were detached and immersed in a solution containing $1 \mathrm{mg} / \mathrm{ml}$ DAB prepared in $\mathrm{HCl}$-acidified ( $\mathrm{pH} \mathrm{3.8)}$ water at $25^{\circ} \mathrm{C}$ for $10 \mathrm{~h}$ and the roots were immersed in DAB solution for $4 \mathrm{~h}$. The samples were then incubated in a boiling solution $(60 \%$ ethanol, $20 \%$ glycerol and $20 \%$ ethylic acid) to wash off green color and then observed and imaged under a DVM6a 3D microscope (Leica, Germany).

233 The OsANN4 coding sequences were cloned into the BD (pGBKT7) vector 
234 between the $N d e \mathrm{I}$ and EcoRI sites with primers P17 and P18. The OsCDPK24

235 (Os11g0171500) coding sequence was cloned into AD (pGADT7) vectors by using 236 primers P19 and P20. The expression vectors pGADT7-OsCDPK24 and 237 pGBKT7-OsANN4 were co-transformed into the yeast strain AH109 via the lithium 238 acetate method (Clontech Yeast Protocols and book). Colonies were then transferred 239 to SM-LWHA and SM-LW. A positive control interaction between the 53 protein and 240 SV40 protein and a negative control interaction between the Lam protein and SV40 241 protein were observed.

242

243

244

245

246

247

\section{Pull down assays}

For induction and purification of OsCDPK24, the plasmid pET28a-OsCDPK24 was induced at $18 \square$ overnight in E. coli strain Rosetta and purified with Ni-NTA Resin. OsANN4-GST and GST were induced at $18 \square$ overnight in E. coli strain Rosetta and purified with glutathione Sepharose 4B beads (GE). Supernatant containing GST or OsANN4-GST was incubated with OsCDPK24-His in $0.5 \mathrm{ml}$ of interaction buffer (25 $\mathrm{mM}$ Tris $\mathrm{pH} 7.2,150 \mathrm{mM} \mathrm{NaCl}$ ) overnight at $4 \square$. GST beads were added and incubated with proteins for $2 \mathrm{~h}$ on a rotating wheel at $4 \square$ followed by washing five times with wash buffer ( $25 \mathrm{mM}$ Tris $\mathrm{pH} 7.2,150 \mathrm{mM} \mathrm{NaCl}, 0.1 \% \mathrm{NP}-40)$. Then, the samples were boiled with $120 \mu \mathrm{l}$ of $1 \times$ SDS loading buffer at $100 \square$ for subsequent western blot analysis.

\section{Luciferase complementation imaging (LCI) assay}

OsANN4 and OsCDPK24 were constructed by using pCAMBIA-Cluc and pCAMBIA-Nluc, respectively, and were transformed into $N$. benthamiana leaves by Agrobacterium strain GV3101. Agrobacterium transformants containing pOsANN4-Cluc, pOsCDPK24-Nluc, empty control pCAMBIA-Cluc and pCAMBIA-Nluc were adjusted to $\mathrm{OD}_{600}=0.5-0.6$ and then injected into tobacco leaves. After spraying the tobacco leaves with $2.5 \mathrm{mM}$ D-luciferin (Goldbio, USA), fluorescent signals were detected and photographed post infiltration by using a Fusion FX7 (Vilber, France) imaging system. The cold luminescence signals values of 
OsANN4 and OsCDPK24 were calculated by Fusion FX7 (Vilber, France).

In vitro kinase assays

Briefly, $5 \mu \mathrm{g}$ of OsANN4-His was incubated with $1.5 \mu \mathrm{g}$ of protein kinase OsCDPK24-His in kinase buffer (20 mM Tris-HCl (pH 7.5), $10 \mathrm{mM} \mathrm{MgCl}, 100 \mathrm{mM}$

$\mathrm{NaCl}, 1 \mathrm{~m} \mathrm{DTT}$ ), and $2 \mathrm{mM}$ ATP was added. The reactions were incubated at $37{ }^{\circ} \mathrm{C}$ for $10 \mathrm{~h}$ and stopped by boiling with $6 \times$ SDS loading buffer. After adding $50 \mathrm{mM}$ Phos-Tag (APExbio, China) and $100 \mathrm{mM} \mathrm{MnCl}_{2}$, the samples were separated by SDS-PAGE and then transferred to nitrocellulose membranes. The signals were detected with a His-tag mouse monoclonal antibody (CWbio, China). Mass spectrometry to detect phosphorylation was finished by Applied Protein Technology Co. Ltd company (Shanghai, China).

\section{Fluorescence measurements of OsANN4}

278 This experiment was finished according to the method described in the study previously (Zhou et al., 2011). $2 \mathrm{mM} \mathrm{Ca}^{2+}$ was added into $2 \mu \mathrm{M}$ recombinant protein, and the fluorescence signals was measured by fluorescence spectrophotometer (F-4600; Hitachi, Japan).

To examine the subcellular distribution of OsANN4, the 35S::OsANN4-GFP vector was constructed. The OsANN4 coding region was fused to GFP using the PMDC83 backbone to construct CaMV35S::OsANN4-GFP, and its expression was driven by the $35 \mathrm{~S}$ promoter. The constructs were introduced into Agrobacterium EHA105 and then transformed into rice calli. Transgenic rice plants expressing OsANN4-GFP were generated as previously described. The signal of OsANN4-GFP protein was examined using confocal laser-scanning microscopy (Zeiss LSM710, Germany). 


\section{RESULTS}

OsANN4 responses to exogenous abscisic acid in rice

We cloned and obtained a putative annexin family gene in rice, OSANN4, which is a ortholog of AtANN4 (Fig. 1A). The genomic sequence of OsANN4 consists of 5 exons and 4 introns, and the open reading frame is predicted to encode a protein with 320 amino acids. Based on sequence searches of the NCBI database, the OsANN4 protein contains three annexin domain architectures, the so-called annexin repeats, which comprise segments of 32, 57, and 67 amino acid residues. Analysis of the 1794-bp promoter sequence upstream of OsANN4 reveals that the OsANN4 promoter contains multiple cis-acting elements that may be associated with abiotic stress (Fig. S1). Then we treated 7-d-old seedlings with $10 \mu \mathrm{M}$ ABA and detected the expression of OsANN4 at $0 \mathrm{~h}, 0.5 \mathrm{~h}, 1 \mathrm{~h}, 3 \mathrm{~h}, 6 \mathrm{~h}, 12 \mathrm{~h}$ and $24 \mathrm{~h}$. The results showed that ABA treatment induced the expression of OSANN4 and the highest expression level after ABA treatment for $6 \mathrm{~h}$, which showed that OsANN4 may respond to exogenous abscisic acid (Fig. 1B).

To test whether OsANN4 is sensitive to $\mathrm{ABA}$ treatment, fifteen independent knocking down OsANN4 transgenic lines were generated. Quantitative real-time PCR analyses showed that OsANN4 was down regulated in all the RNAi lines (Fig. 1D). The homozygous plants of R4, R12 and R15 showed obviously decreased transcriptional level of OsANN4 and to be used for the further analyzed. Seeds from a wild-type (WT) line and three RNAi lines (R4, R12 and R15) were planted on 1/2 Murashige and Skoog (MS) medium supplemented with $10 \mu \mathrm{M}$ ABA, and on 1/2 MS medium as a control. Twelve days later, both WT and RNAi plants showed no significant difference at the stage of early seedling growth without ABA treatment (Supplementary Fig. S2A). Otherwise, the shoots of RNAi lines were shorter than WT with ABA (Fig. 1C, E). Furthermore, the length of the root and the numbers of adventitious roots were not significantly different from that of the wild type after $\mathrm{ABA}$ treatment, whereas the number of lateral roots was significantly less relative to wild type line (Fig. 1E, F). This result indicates that the RNAi seedlings are sensitive to $\mathrm{ABA}$ in the early seedling stage. 
To understand if OsANN4 expression is involved in ABA response, we further examined the expression levels of OsANN4 and several genes related ABA biosynthesis and response in RNAi lines and WT treated with $10 \mu \mathrm{M}$ ABA for $0 \mathrm{~h}$, $0.5 \mathrm{~h}, 1 \mathrm{~h}, 3 \mathrm{~h}$ and $6 \mathrm{~h}$. The expression levels of OsANN4 in RNAi lines were always lower relative to WT (Supplementary Fig. S2B). Under both normal and ABA treatment, OsNCED4 and RAB16A expression were decreased in the RNAi lines, especially in 6h (Supplementary Fig. S2C, S2E). However, the expression of the OsZEP transcripts did not differ between the WT and RNAi lines (Supplementary Fig. $\mathrm{S} 2 \mathrm{D})$. These data sufficiently demonstrated that OSANN4 is closely related to ABA response.

\section{OsANN4 modulates ROS in response to ABA in rice roots}

More evidence suggests that ABA-enhanced stress tolerance is associated with the induction of the antioxidant defense system to protect plant cells against oxidative damage (Xing et al., 2008; Ozfidan et al., 2012). SOD and CAT may protect plant cells from oxidative damage by eliminating ROS, which are important signaling molecules in the ABA pathway. SOD is a critical enzyme that catalyzes $\mathrm{O}_{2}{ }^{-}$to $\mathrm{H}_{2} \mathrm{O}_{2}$, and CAT is a key enzyme that catalyzes $\mathrm{H}_{2} \mathrm{O}_{2}$ to $\mathrm{H}_{2} \mathrm{O}$ and $\mathrm{O}_{2}$ which protects plant cells from oxidative damage by eliminating ROS. To assess the effect of knocking down OSANN4 on antioxidant defense to ABA response, we detected the activities of SOD and CAT in 7-d-old seedlings without or with $10 \mu \mathrm{M}$ ABA treatment for $1 \mathrm{~h}$. The RNAi lines showed no significant difference in activities of both SOD and CAT relative to those of the WT without treatment. After ABA treatment for $1 \mathrm{~h}$, both the SOD and CAT activities of RNAi lines were decreased relative to WT (Fig. 2A, B).

We further detected $\mathrm{H}_{2} \mathrm{O}_{2}$ content in 7-d-old RNAi and WT seedlings. The RNAi lines showed increased $\mathrm{H}_{2} \mathrm{O}_{2}$ content in both shoots and roots after $10 \mu \mathrm{M} A B A$ treatment (Fig. 2C, D). The results were consistent with SOD and CAT activity. Next, we assayed the production of $\mathrm{H}_{2} \mathrm{O}_{2}$ in situ of RNAi lines and WT. Seven-day-old rice seedlings were treated with or without $10 \mu \mathrm{M} A B A$ for $5 \mathrm{~h}$, roots and leaves were then 
subjected to 3, 3'-diaminobenzidine (DAB) staining respectively. Without ABA treatment, there were no visible differences in rice roots and leaves of WT and RNAi lines. However, increased numbers of darkly stained patches were observed in mesophyll cells and root cells of RNAi lines (Fig. 2E, F), indicating an increased level of $\mathrm{H}_{2} \mathrm{O}_{2}$ in these plants.

\section{OsANN4 promotes stomatal closure and alleviates $\mathrm{H}_{2} \mathrm{O}_{2}$-induced aerenchyma} formation with ABA treatment

It is reported that a certain ROS content resulted in programmed cell death and further promoted aerenchyma formation (Yamauchi et al., 2017). The $\mathrm{H}_{2} \mathrm{O}_{2}$ content was obviously increased in the roots of the OSANN4-RNAi lines, then we investigate whether OsANN4 affects aerenchyma formation in adventitious roots. Twelve-day-old OsANN4-RNAi lines as well as WT were planted in 1/2 MS medium supplemented with $10 \mu \mathrm{M}$ ABA. Upon analysis of the transverse sections, aerenchyma formation was observed only in the RNAi lines and not in the WT at 3-mm from root tips. Furthermore, aerenchyma formation was observed in both RNAi lines and WT at the 5-mm transverse sections (Fig. 3A, B). Then we also detected $\mathrm{H}_{2} \mathrm{O}_{2}$ content of 12-d-old RNAi and WT seedlings with $10 \mu \mathrm{M}$ ABA treatment. The RNAi lines showed higher $\mathrm{H}_{2} \mathrm{O}_{2}$ content relative to WT with ABA treatment, which may result in an increase in the number of aerenchyma in the RNAi lines (Supplementary Fig. S3).

We further assessed the stomatal apertures of these plants using scanning electron microscopy images of rice leaves. The proportion of stomatal closure in WT was significantly increased with $\mathrm{ABA}$ treatment than that without $\mathrm{ABA}$ treatment. However, for RNAi plants, the statistical showed no significantly differences of stomatal openness and closure with or without $10 \mu \mathrm{M}$ ABA treatment (Fig. 3C, D). It suggests that OsANN4 may play a key role in ABA-induced stomatal closure.

\section{OsANN4 interacts with the protein kinase OsCDPK24}

In previous reports, annexins were shown to interact with protein kinases, including SAPKs and CDPKs (Rohila et al., 2006; Qiao et al., 2015). To further understand how 
OsANN4 response to ABA, we used yeast two-hybrid assay to verify several potential rice protein kinase candidates, including Os01g0570500, Os10g0518800, Os01g0869900, Os11g0171500. With the results, we did not find Os01g0570500 and Os10g0518800 interacted with OsANN4 separately. However, Os01g0869900, belongs to sucrose nonfermenting1-related protein kinase2 (SnRK2) family, showed weak interaction with OsANN4. Furthermore, OsCDPK24 (Os11g0171500), a key regulator in response to $\mathrm{ABA}$ (Wang et al., 2016), showed strong interaction with OsANN4 (Fig. 4A).

To further verify the interactions between OsANN4 and OsCDPK24, the pull-down system in vitro was carried out. OsCDPK24-His and OsANN4-GST recombinant proteins were induced in Escherichia coli and purified to perform the pull-down assay. OsANN4-GST could pull down OsCDPK24-His, whereas GST could not, it was further proved the interaction between OsANN4 and OsCDPK24 (Fig. 4B).

We also obtained additional confirmation of the interaction between OsANN4 and OsCDPK24 in Nicotianana benthamiana leaves by using a luciferase complementation imaging (LCI) assay. After spraying D-luciferin to tobacco leaves, a fluorescent signal occurred when $\mathrm{p} O s A N N 4$-Cluc and $\mathrm{p} O s C D P K 24-\mathrm{Nluc}$ were present simultaneously, which showed that OsANN4 can interact with OsCDPK24 (Fig. 4C).

\section{The 13th serine residue is a key phosphorylation site for OsANN4}

To understand the mechanism underlying the interaction of OsANN4 and OsCDPK24, we performed a phosphorylation assay in vitro to determine whether OsANN4 is a substrate of OsCDPK24. The Phos-tag reagent was used to separate phosphorylated proteins from non-phosphorylated proteins according to their different migration rates. When purified OsANN4-His and OsCDPK24-His were incubated together, phosphorylated OsANN4 bands were detectable with a His-tag antibody. The phosphorylation level of OsANN4 increased slightly after the addition of $5 \mu \mathrm{M}$ and $500 \mu \mathrm{M} \mathrm{Ca}^{2+}$. These results indicated that OsANN4 can be phosphorylated by OsCDPK24, especially in the presence of $\mathrm{Ca}^{2+}$ (Fig. 5A).

To further demonstrate that OsANN4 was a substrate of OsCDPK24, mass 
411 spectrometry was performed to detect specific phosphorylation sites of OsANN4

412 when OsCDPK24 was present. Mass spectrometry analysis showed that OsANN4 can

413 be phosphorylated by OsCDPK24, and the OsANN4 phosphorylation site was the

414 13th amino acid, which is a serine (Fig. 5B).

415

416

417

418

419

420

421

422

423

The interaction of OSANN4 and OSCDPK24 plays a role in the response to ABA

To determine whether the interaction of OsANN4 and OsCDPK24 plays a role in the response to $\mathrm{ABA}$, we performed $10 \mu \mathrm{M} \mathrm{ABA}$ treatment on tobacco leaves injected with Agrobacterium GV3101 containing pOsANN4-Cluc and pOsCDPK24-Nluc. After ABA treatment, LUC cold luminescence signals increased significantly, which demonstrated the interaction between OsANN4 and OsCDPK24 was enhanced with ABA treatment (Fig. 6A and B). These results indicated that OsANN4 interacted with OsCDPK24, and this interaction increased under $\mathrm{ABA}$ treatment, thus, the OsANN4-OsCPK24 interaction may play a role in the response to ABA in rice.

To demonstrate whether the phosphorylation of OsANN4 plays a role in response to ABA signaling, we mutated the serine $(\mathrm{S})$ residue with non-phosphorylatable alanine (A) to inhibit the phosphorylation of OsANN4 and constructed the pOsANN4 (S13A)-Cluc vector to perform an LCI assay. We found that after mutation of phosphorylation site in OsANN4, OsANN4 still can interact with OsCDPK24. After spraying with ABA, the responses of OsANN4 (S13A)-Cluc and OsCDPK24-Nluc to ABA were significantly weakened (Fig. 6C, D). This suggests that this key phosphorylation site plays an important role in OsANN4 response to ABA.

\section{OsANN4 is a $\mathrm{Ca}^{2+}$-binding protein that locates to the cell periphery}

Previous studies have shown that the subcellular localization of annexin may be altered due to environmental stimuli (Baucher et al., 2012; Qiao et al., 2015). To explore whether OsANN4 altered its subcellular localization with ABA treatment, 35Spro::OsANN4-GFP was introduced into Agrobacterium EHA105 and then transformed the rice callus. Twenty-five independent transgenic lines were obtained, and 3 lines were used for further analyses. We observed OsANN4 was located to the 
cell periphery by a confocal laser-scanning microscope (Zeiss LSM710). After $10 \mu \mathrm{M}$ ABA treatment, the signal of OsANN4-GFP remained unchanged (Supplementary Fig. S4).

Annexins are considered to be a class of protein interacting with biological membranes in a calcium dependent or independent manner. In this study, we detected the $\mathrm{Ca}^{2+}$ binding activity of OsANN4. The fluorescence level of OsANN4-His recombinant protein was determined by fluorescence spectrophotometer (F-4600; Hitachi, Japan). Upon excitation at $315 \mathrm{~nm}$ (Fig. 5C), the fluorescence emission spectrum showed the maximum fluorescence wavelength $(\lambda \max )$ at $390 \mathrm{~nm}$, where the fluorescence intensity reached approximately 2000 A.U.. The maximum fluorescence intensity of the OsANN4-His recombinant protein was measured again after the addition of $2 \mathrm{mM} \mathrm{Ca}{ }^{2+}$. The results showed that the maximum fluorescence wavelength remained unchanged, whereas the fluorescence intensity changed (Fig. $5 \mathrm{E}$ ), indicating that the OsANN4 has $\mathrm{Ca}^{2+}$ binding activity and may further change the conformation of the protein.

Since the phosphorylation site of OsANN4 plays a role in the ABA response, we examined whether this phosphorylation site affects its binding to $\mathrm{Ca}^{2+}$. OsANN4 (S13A)-His recombinant protein was induced and purified to perform the above fluorescence assay. The results showed that upon excitation at $315 \mathrm{~nm}$ (Fig. 5D), the fluorescence emission spectrum of OsANN4 (S13A)-His recombinant protein remained unchanged whereas the fluorescence level has changed (Fig. 5F), which indicated that the phosphorylation site of OsANN4 does not affect its binding to $\mathrm{Ca}^{2+}$ but may change the conformation of OsANN4.

\section{Discussion}

\section{OsANN4 modulates ROS production to alleviate aerenchyma formation}

Recent results have revealed that annexins are involved in the responses to various abiotic stresses including salt, drought, oxidative and other stresses (Clark et al., 1998; Lee et al., 2004; Clark et al., 2012; Jami et al., 2012; Richards et al., 2014; Qiao et al., 2015). Evidence has shown that induction of the antioxidant defense system protects 
plant cells against oxidative damage (Mittler et al., 2011; Richards et al., 2014; Del Rio, 2015; Qiao et al., 2015). And ROS are important signaling molecules, especially in the ABA signaling pathway. Herein, we reported OsANN4, a ortholog of AtANN4, modulates $\mathrm{H}_{2} \mathrm{O}_{2}$ accumulation to alleviate root aerenchyma formation with exogenous abscisic acid. Our results showed that knocking down OsANN4 decreased the activity of SOD and CAT with ABA treatment, and more $\mathrm{H}_{2} \mathrm{O}_{2}$ content was measured (Fig. 2), further resulted in earlier aerenchyma formation (Fig. 3A, B). We conclude that OsANN4 promotes redox reactions, potentially regulating SOD and CAT to scavenge $\mathrm{H}_{2} \mathrm{O}_{2}$.

Hydrogen peroxide $\left(\mathrm{H}_{2} \mathrm{O}_{2}\right)$, is also an essential secondary messenger to be involved in ABA-induced stomatal closure. Interestingly, in our study, a high level of $\mathrm{H}_{2} \mathrm{O}_{2}$ content did not result in a high proportion of stomatal closure in OsANN4-RNAi lines (Fig. 3D). Abscisic acid causes the $\mathrm{Ca}^{2+}$ concentration in cytoplasmic to elevate in guard cells via $\mathrm{Ca}^{2+}$ influx, results in stomatal closure (Hedrich, 2016; Kong et al., 2016). We speculate that OsANN4, a putative $\mathrm{Ca}^{2+}$ channel, may function in stomatal closure by affecting $\mathrm{Ca}^{2+}$ influx under $\mathrm{ABA}$ treatment.

\section{OsANN4 mediates $\mathrm{Ca}^{2+}$ and interacts with OsCDPK24 in response to ABA}

In rice, a previous study showed that a rice calcium-dependent protein kinase OsCDPK12 can induce expression of the antioxidant genes $O s A P X 2$ and $O s A P X 8$ under salt stress and reduce the salt-induced accumulation of $\mathrm{H}_{2} \mathrm{O}_{2}$ (Asano et al., 2012), suggesting that $O s C D P K 12$ positively regulates ROS detoxification by controlling the expression of antioxidant genes. ZmCPK11 has been proven to be involved in ABA-induced antioxidant defense and to function upstream of ZmMPK5 in ABA signaling in maize (Ma et al., 2016). However, the regulatory network regulating antioxidant defense in ABA signaling remains to be elucidated. In this study, we demonstrated that OsANN4 interacts with the kinase OsCDPK24 (Fig.4), and furthermore, this interaction plays a role in the ABA response (Fig. 6). Moreover, OsANN4 is a substrate for OsCDPK24 and OsANN4 phosphorylation site is the 13th serine (Fig. 5), which is a key site for phosphorylation. After mutated the serine 
residue with non-phosphorylatable alanine (A) to inhibit the phosphorylation of OsANN4, the conformation of OsANN4 has changed (Fig. 5F) and the interaction of OsANN4 and OsCDPK24 was weakened (Fig. 6C, D), indicating that under ABA treatment, OsCDPK24 may be more inclined to interact with the phosphorylated OsANN4, rather than the non-phosphorylated state. RCAR is a component of the $\mathrm{ABA}$ receptor complex, and $\mathrm{PP} 2 \mathrm{C}$ phosphatases inhibit the activity of some kinases, similar to OsCDPK, which can be dephosphorylated and promote ion channel expression (Kudla et al., 2018) in response to ABA. However, whether CDPKs are involved in $\mathrm{ABA}$-induced antioxidant defense remains to be determined.

Annexins are traditionally perceived as $\mathrm{Ca}^{2+}$-dependent phospholipid-binding proteins, which usually contain a characteristic type $\mathrm{II} \mathrm{Ca}^{2+}$-binding residue in each corresponding repeat in vertebrates. In plant annexins, the type II $\mathrm{Ca}^{2+}$-binding residues are absent in repeats 2 and 3, and no type $\mathrm{II} \mathrm{Ca}^{2+}$-binding residues are found in OsANN4 (predicted at ScanProsite http://ca.expasy.org/tools/scanprosite/).

However, our results indicate that OsANN4 has the $\mathrm{Ca}^{2+}$ binding activity, which could be perceived as evidence that annexins can also function in their $\mathrm{Ca}^{2+}$-free conformation in an unknown intricate fashion, thus increasing the functional diversity of annexins. After mutating the phosphorylation site of OsANN4, the protein conformation of OsANN4 is altered, but it still can bind to $\mathrm{Ca}^{2+}$ (Fig. 5F). What's more, some data have indicated that ion channels or transporters act as targets of $\mathrm{Ca}^{2+}$ signaling networks (Whalley et al., 2011; Edel and Kudla, 2015; Kudla et al., 2018). As a putative ion channel, OsANN4 can interact with OsCDPK24, supporting the existence of potential connection of the annexin, calcium and protein kinase in response to stress.

\section{A possible modle for OsANN4 to respond to ABA}

The conformation of PYL can be changed after binding ABA, thus enhancing the stability of the complexes of PYL and PP2C, resulting in a reduced inhibitory effect of PP2C on kinases (SnRK or CDPK, etc.), which then activates transcription factors, ion channels or other ABA-responsive genes (Finkelstein, 2013; Kudla et al., 2018; 
531 Wang et al., 2018). Based on our experimental results, we proposed a working model 532 for OsANN4 functioning as a key protein in modulating $\mathrm{H}_{2} \mathrm{O}_{2}$ accumulation to 533 alleviate root aerenchyma formation with exogenous abscisic acid (Fig. 7). OsANN4

534 is a $\mathrm{Ca}^{2+}$-binding protein, and it can interact with and be phosphorylated by 535 OsCDPK24 at the 13th serine. When ABA is present, PP2C binds to the PYL receptor, 536 may result in a decrease in the inhibitory effect of PP2C on OsCDPK24. The 537 interaction between OsANN4 and OsCDPK24 is enhanced to response ABA. When 538 OsCDPK24 interacts with OsANN4, it may be more inclined to phosphorylate 539 OsANN4 under ABA treatment. OsANN4 promotes $\mathrm{O}_{2}{ }^{--}$to $\mathrm{H}_{2} \mathrm{O}_{2}$ by increasing SOD 540 activity, then converts $\mathrm{H}_{2} \mathrm{O}_{2}$ into $\mathrm{H}_{2} \mathrm{O}$ through CAT activity, thereby reducing ROS 541 content $\left(\mathrm{O}_{2}^{-}\right.$and $\left.\mathrm{H}_{2} \mathrm{O}_{2}\right)$. As a consequence, OsANN4 can further alleviate 542 programmed cell death and inhibit the formation of aerenchyma.

\section{Supplementary data}

Supplementary data are available at JXB online.

Fig. S1. Analysis of cis-acting elements in the promoter of OsANN4.

Fig. S2. The relative transcript levels of OsANN4, OsZEP, OsNCED4 and OsRAB16A in WT and OsANN4-R plants with ABA treatment

Fig. S3. The $\mathrm{H}_{2} \mathrm{O}_{2}$ content in the roots of 12-d-old RNAi and WT seedlings planted in $1 / 2 \mathrm{MS}$ medium supplemented with or without $10 \mu \mathrm{M} \mathrm{ABA}$.

Fig. S4. Subcellular localization of OsANN4-GFP in the 3-day-old root tips cell with or without ABA treatmentl.

553 Table S1. Primer sequences used in plasmid construction and qPCR.

\section{Acknowledgements}

556 This work was supported by the National Natural Science Foundation of China

557 (31571638, 31340046), Research Fund for the Doctoral Program of Higher Education 558 (20131303110005) and the Advanced Postdoctoral Science Programs Foundation of 559 He'bei Educational Committee (B2016003012). 
We thank Dr. Kang Chong of the Institute of Botany, Chinese Academy of Science,

Co. Ltd for the technical assistance in LC/MS proteomic assays.

\section{References}

Asano T, Hayashi N, Kobayashi M, et al. 2012. A rice calcium-dependent protein kinase OsCDPK12 oppositely modulates salt-stress tolerance and blast disease resistance. Plant J 69, 26-36

Baucher, M, Perez-Morga, D, and El Jaziri, M. 2012. Insight into plant annexin function, from shoot to root signaling. Plant signaling \& behavior 7, 524-528.

568 Bueso E, Rodriguez L, Lorenzo-Orts L, Gonzalez-Guzman M, Sayas E, Munoz-Bertomeu J, 569 Ibanez C, Serrano R, Rodriguez PL. 2014. The single-subunit RING-type E3 ubiquitin ligase RSL1 570 targets PYL4 and PYR1 ABA receptors in plasma membrane to modulate abscisic acid signaling. Plant J 80, 1057-1071

572 Campbell R, Drew MC. 1983. Electron microscopy ofgas space (aerenchyma) formation in 573 adventitious roots of Zea mays L. subjected to oxygen shortage. Planta 157, 350-357

574 Clark GB, Dauwalder M, Roux SJ. 1998. Immunological and biochemical evidence for nuclear localization of annexin in peas. Plant Physiol Biochem 36, 621-627 Clark GB, Morgan RO, Fernandez MP, Roux SJ. 2012. Evolutionary adaptation of plant annexins has diversified their molecular structures, interactions and functional roles. New Phytol 196, 695-712 Colmer TD. 2003. Aerenchyma and an inducible barrier to radial oxygen loss facilitate root aeration in 579 upland, paddy and deep-water rice (Oryza sativa L.). Ann Bot 91 Spec No, 301-309

580 Colmer TD, Pedersen O. 2008. Oxygen dynamics in submerged rice (Oryza sativa). New Phytol. 178, $581 \quad 326-334$.

582 Cutler SR, Rodriguez PL, Finkelstein RR, Abrams SR. 2010. Abscisic acid: emergence of a core signaling network. Annu Rev Plant Biol 61, 651-679

584 Del Rio LA. 2015. ROS and RNS in plant physiology: an overview. J Exp Bot 66, 2827-2837

585 Ding Y, Cao J, Ni L, Zhu Y, Zhang A, Tan M, Jiang M. 2013. ZmCPK11 is involved in abscisic 586 acid-induced antioxidant defence and functions upstream of ZmMPK5 in abscisic acid signalling in 587 maize. J Exp Bot 64, 871-884

588 Edel KH, Kudla J. 2015. Increasing complexity and versatility: how the calcium signaling toolkit was 589 shaped during plant land colonization. Cell Calcium 57: 231-246

590 Finkelstein,R. 2013. Abscisic acid synthesis and response. The Arabidopsis Book 11, e0166-e0166

591 Fujii H, Chinnusamy V, Rodrigues A, Rubio S, Antoni R, Park SY, Cutler SR, Sheen J, Rodriguez

592 PL, Zhu JK. 2009. In vitro reconstitution of an abscisic acid signalling pathway. Nature 462, 660-664

593 Giraudat J, Parcy F, Bertauche N, Gosti F, Leung J, Morris PC, Bouvier-Durand M, Vartanian N.

594 1994. Current advances in abscisic acid action and signalling. Plant Mol Biol 26, 1557-1577

595 Hedrich R. 2012. Ion channels in plants. Physiol Rev 92, 1777-1811

596 He Y, Hao Q, Li W, Yan C, Yan N, Yin P. 2014. Identification and characterization of ABA receptors 597 in Oryza sativa. PLoS One $\mathbf{9}$, e95246

598 Himmelbach A, Yang Y, Grill E. 2003. Relay and control of abscisic acid signaling. Curr Opin Plant 599 Biol 6, 470-479

600 Jami SK, Clark GB, Ayele BT, Roux SJ, Kirti PB. 2012. Identification and characterization of 
601

602

603

604

605

606

607

608

609

610

611

612

613

614

615

616

617

618

619

620

621

622

623

624

625

626

627

628

629

630

631

632

633

634

635

636

637

638

639

640

641

642

643

644

annexin gene family in rice. Plant Cell Rep 31, 813-825

Jiang M, Zhang J. 2002. Water stress-induced abscisic acid accumulation triggers the increased generation of reactive oxygen species and up-regulates the activities of antioxidant enzymes in maize leaves. J Exp Bot 53, 2401-2410

Joshi R, Kumar P. 2012. Lysigenous aerenchyma formation involves non-apoptotic programmed cell death in rice (Oryza sativa L.) roots. Physiol Mol Biol Plants 18, 1-9

Klingler JP, Batelli G, Zhu JK. 2010. ABA receptors: the START of a new paradigm in phytohormone signalling. J Exp Bot 61, 3199-3210

Kobayashi M, Ohura I, Kawakita K, Yokota N, Fujiwara M, Shimamoto K, Doke N, Yoshioka H. 2007. Calcium-dependent protein kinases regulate the production of reactive oxygen species by potato NADPH oxidase. Plant Cell 19, 1065-1080

Kong DD, Hu HC, Okuma E, et al. 2016. L-Met activates Arabidopsis GLR $\mathrm{Ca}^{2+}$ channels upstream of ROS production and regulates stomatal movement. Cell Reports 17, 2553-2561

Kovacs I, Ayaydin F, Oberschall A, Ipacs I, Bottka S, Pongor S, Dudits D, Toth EC. 1998. Immunolocalization of a novel annexin-like protein encoded by a stress and abscisic acid responsive gene in alfalfa. Plant J 15, 185-197

Kudla J, Becker D, Grill E, Hedrich R, Hippler M, Kummer U, Parniske M, Romeis T, Schumacher K. 2018. Advances and current challenges in calcium signaling. New Phytol 218, 414-431

Laohavisit A, Brown AT, Cicuta P, Davies JM. 2010. Annexins: components of the calcium and reactive oxygen signaling network. Plant Physiol 152, 1824-1829

Lee S, Lee EJ, Yang EJ, Lee JE, Park AR, Song WH, Park OK. 2004. Proteomic identification of annexins, calcium-dependent membrane binding proteins that mediate osmotic stress and abscisic acid signal transduction in Arabidopsis. Plant Cell 16, 1378-1391

Licausi F. 2013. Molecular elements of low-oxygen signaling in plants. Physiol Plant 148, 1-8

Licausi F, Kosmacz M, Weits DA, Giuntoli B, Giorgi FM, Voesenek LA, Perata P, van Dongen JT. 2011. Oxygen sensing in plants is mediated by an N-end rule pathway for protein destabilization. Nature 479, 419-422

Liu J, Zhang C, Wei C, Liu X, Wang M, Yu F, Xie Q, Tu Jumin. 2016. The ring finger ubiquitin E3 ligase OsHTAS enhances heat tolerance by promoting $\mathrm{H}_{2} \mathrm{O}_{2}$-induced stomatal closure in rice. Plant Physiology, 170(1), 429

Liu Y, Xu C, Zhu Y, Zhang L, Chen T, Zhou F, Chen H, Lin Y. 2018. The calcium-dependent kinase OsCPK24 functions in cold stress responses in rice. J Integr Plant Biol 60, 173-188

Ma F, Ni L, Liu L, Li X, Zhang H, Zhang A, Tan M, Jiang M. 2016. ZmABA2, an interacting protein of ZmMPK5, is involved in abscisic acid biosynthesis and functions. Plant Biotechnol J 14, 771-782

Ma Y, Szostkiewicz I, Korte A, Moes D, Yang Y, Christmann A, Grill E. 2009. Regulators of PP2C phosphatase activity function as abscisic acid sensors. Science 324, 1064-1068

McCourt P, Creelman R. 2008. The ABA receptors -- we report you decide. Curr Opin Plant Biol 11, 474-478

Melcher K, Zhou XE, Xu HE. 2010. Thirsty plants and beyond: structural mechanisms of abscisic acid perception and signaling. Curr Opin Struct Biol 20, 722-729

Miao Y, Lv D, Wang P, Wang XC, Chen J, Miao C, Song CP. 2006. An Arabidopsis glutathione peroxidase functions as both a redox transducer and a scavenger in abscisic acid and drought stress responses. Plant Cell 18, 2749-2766 
645 Miller G, Suzuki N, Ciftci-Yilmaz S, Mittler R. 2010. Reactive oxygen species homeostasis and 646 signalling during drought and salinity stresses. Plant Cell Environ 33, 453-467

647 Mittler R, Vanderauwera S, Suzuki N, Miller G, Tognetti VB, Vandepoele K, Gollery M, Shulaev

648 V, Van Breusegem F. 2011. ROS signaling: the new wave? Trends Plant Sci 16, 300-309

649 Moss SE, Morgan RO. 2004. The annexins. Genome Biol 5, 219

650 Neill S, Barros R, Bright J, Desikan R, Hancock J, Harrison J, Morris P, Ribeiro D, Wilson I.

651 2008. Nitric oxide, stomatal closure, and abiotic stress. J Exp Bot 59, 165-176

652 Ng LM, Melcher K, Teh BT, Xu HE. 2014. Abscisic acid perception and signaling: structural 653 mechanisms and applications. Acta Pharmacol Sin 35, 567-584

654 Nishimura N, Sarkeshik A, Nito K, et al. 2010. PYR/PYL/RCAR family members are major in-vivo 655 ABI1 protein phosphatase 2C-interacting proteins in Arabidopsis. Plant J 61, 290-299

656 Ni L, Fu X, Zhang H, et al. 2018. Abscisic acid inhibits rice protein phosphatase PP45 via $\mathrm{H}_{2} \mathrm{O}_{2}$ and

657 relieves repression of the $\mathrm{Ca}^{2+} / \mathrm{CaM}$-dependent protein kinase DMI3. Plant Cell 658 doi:10.1105/tpc.18.00506

659 Ozfidan C, Turkan I, Sekmen AH, Seckin B. 2012. Abscisic acid-regulated responses of aba2-1

660 under osmotic stress: the abscisic acid-inducible antioxidant defence system and reactive oxygen

661 species production. Plant Biol (Stuttg). 14, 337-346

662 Park SY, Fung P, Nishimura N, et al. 2009. Abscisic acid inhibits type 2C protein phosphatases via 663 the PYR/PYL family of START proteins. Science 324, 1068-1071

664 Pekic S, Stikic R, Tomljanovic L, Andjelkovic V, Ivanovic M, Quarrie SA. 1995. Characterization 665 of maize lines differing in leaf abscisic acid content in the field .1. abscisic acid physiology. Ann Bot $66675,67-73$

667 Qiao B, Zhang Q, Liu D, et al. 2015. A calcium-binding protein, rice annexin OsANN1, enhances heat 668 stress tolerance by modulating the production of $\mathrm{H}_{2} \mathrm{O}_{2}$. J Exp Bot 66, 5853-5866

669 Qu L, Wu C, Zhang F, Wu Y, Fang C, Jin C, Liu X, Luo J. 2016. Rice putative methyltransferase 670 gene OsTSD2 is required for root development involving pectin modification. J Exp Bot 67, 5349-5362

671 Rajhi I, Yamauchi T, Takahashi H, et al. 2011. Identification of genes expressed in maize root

672 cortical cells during lysigenous aerenchyma formation using laser microdissection and microarray 673 analyses. New Phytol 190, 351-368.

674 Rahoui S, Martinez Y, Sakouhi L, Ben C, Rickauer M, El Ferjani E, Gentzbittel L, Chaoui A. 675 2017. Cadmium-induced changes in antioxidative systems and differentiation in roots of contrasted 676 Medicago truncatula lines. Protoplasma 254, 473-489

677 Richards SL, Laohavisit A, Mortimer JC, Shabala L, Swarbreck SM, Shabala S, Davies JM. 2014. 678 Annexin 1 regulates the $\mathrm{H}_{2} \mathrm{O}_{2}$-induced calcium signature in Arabidopsis thaliana roots. Plant $\mathrm{J} 77$, 679 136-145

680 Rohila JS, Chen M, Chen S, et al. 2006. Protein-protein interactions of tandem affinity 681 purification-tagged protein kinases in rice. Plant J 46, 1-13

682 Santiago J, Rodrigues A, Saez A, Rubio S, Antoni R, Dupeux F, Park SY, Marquez JA, Cutler SR, 683 Rodriguez PL. 2009. Modulation of drought resistance by the abscisic acid receptor PYL5 through 684 inhibition of clade A PP2Cs. Plant J 60, 575-588

685 Shiono K, Ogawa S, Yamazaki S, Isoda H, Fujimura T, Nakazono M, Colmer TD. 2011. 686 Contrasting dynamics of radial $\mathrm{O}_{2}$-loss barrier induction and aerenchyma formation in rice roots of two 687 lengths. Ann Bot 107, 89-99 
Umezawa T, Nakashima K, Miyakawa T, Kuromori T, Tanokura M, Shinozaki K, Yamaguchi-Shinozaki K. 2010. Molecular basis of the core regulatory network in ABA responses: sensing, signaling and transport. Plant Cell Physiol 51, 1821-1839

Wang L, Yu C, Xu S, Zhu Y, Huang W. 2016. OsDi19-4 acts downstream of OsCDPK14 to positively regulate ABA response in rice. Plant Cell Environ 39, 2740-2753

Wang P, Song CP. 2008. Guard-cell signalling for hydrogen peroxide and abscisic acid. New Phytol 178, 703-718

Wang P, Zhao Y, Li Z, et al. 2018. Reciprocal Regulation of the TOR Kinase and ABA Receptor Balances Plant Growth and Stress Response. Molecular Cell 69(1), 100-112

Whalley HJ, Sargeant AW, Steele JF, Lacoere T, Lamb R, Saunders NJ, Knight H, Knight MR. 2011. Transcriptomic analysis reveals calcium regulation of specific promoter motifs in Arabidopsis. Plant Cell 23, 4079-4095

Xing Y, Jia W, Zhang J. 2008. AtMKKI mediates ABA-induced CATl expression and $\mathrm{H}_{2} \mathrm{O}_{2}$ production via AtMPK6-coupled signaling in Arabidopsis. Plant J 54, 440-451

Yamauchi T, Watanabe K, Fukazawa A, Mori H, Abe F, Kawaguchi K, Oyanagi A, Nakazono M. 2014. Ethylene and reactive oxygen species are involved in root aerenchyma formation and adaptation of wheat seedlings to oxygen-deficient conditions. J Exp Bot 65, 261-273.

Yamauchi T, Yoshioka M, Fukazawa A, Mori H, Nishizawa NK, Tsutsumi N, Yoshioka H, Nakazono M. 2017. An NADPH oxidase RBOH functions in rice roots during lysigenous aerenchyma formation under oxygen-deficient conditions. Plant Cell 29, 775-790

Ye Y, Zhou L, Liu X, Liu H, Li D, Cao M, Chen H, Xu L, Zhu JK, Zhao Y. 2017. A novel chemical inhibitor of ABA signaling targets all ABA receptors. Plant Physiol 173, 2356-2369

Zhao Y, Chan Z, Xing L, Liu X, Hou YJ, Chinnusamy V, Wang P, Duan C, Zhu JK. 2013. The unique mode of action of a divergent member of the ABA-receptor protein family in ABA and stress signaling. Cell Res 23, 1380-1395

Zhou L, Duan J, Wang XM, Zhang HM, Duan MX, Liu JY. 2011. Characterization of a novel annexin gene from cotton (Gossypium hirsutum cv CRI 35) and antioxidative role of its recombinant protein. J Integr Plant Biol 53, 347-357

Zhu S, Yu X, Wang X, et al. 2007. Two calcium-dependent protein kinases, CPK4 and CPK11, regulate abscisic acid signal transduction in Arabidopsis. Plant Cell 19, 3019-3036 
731

732

733

734

735

736

737

738

739

740

741

742

743

744

745

746

747

748

749

750

751

752

753

754

755

756

757

758

759

760

761

Fig. 1. OsANN4 responses to exogenous abscisic acid in rice.

A Phylogenic tree of OsANN4 and the Arabidopsis orthologue AtANNs. B OsANN4 transcript expression levels under ABA treatment. C WT and OsANN4 RNAi lines were grown for $12 \mathrm{~d}$ in $10 \mu \mathrm{M}$ ABA medium. The figure shows the representative results of five replicates with $\mathrm{T} 2$ generation rice. Scale bars are $1 \mathrm{~cm}$. D Relative transcript levels of OSANN4 in OsANN4 RNAi transgenic rice.

E. The length of shoots and roots in 12-d-old WT and OsANN4 RNAi plants with $10 \mu \mathrm{M} \mathrm{ABA}$ treatment. F The numbers of adventitious roots and lateral roots in 12-d-old WT and OsANN4 RNAi lines with $10 \mu \mathrm{M}$ ABA treatment. Values represent the mean \pm SD from three independent experiments. Statistical significance was determined by Student's t-test, ${ }^{*} \mathrm{P}<0.05, * * \mathrm{P}<0.01, * * * \mathrm{P}<0.001$.

(1)

6

(1)

8

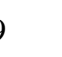

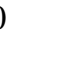


Fig. 2. OsANN4 modulates ROS in response to $\mathrm{ABA}$ in rice roots

766 A SOD activities in 7-d-old WT and OsANN4-R plants with or without $10 \mu \mathrm{M}$ ABA treatment for1h. B

767 CAT activities in 7-d-old WT and OsANN4-R plants with or without $10 \mu \mathrm{M}$ ABA treatment for $1 \mathrm{~h}$. C

768 The $\mathrm{H}_{2} \mathrm{O}_{2}$ content in the shoots of 7-d-old WT and OsANN4-R plants with or without $10 \mu \mathrm{M}$ ABA

769 treatment for $1 \mathrm{~h}$. D The $\mathrm{H}_{2} \mathrm{O}_{2}$ content in the roots of 7-d-old WT and OsANN4-R plants with or without

$10 \mu \mathrm{M}$ ABA treatment for 1 h. E Histochemical detection of $\mathrm{H}_{2} \mathrm{O}_{2}$ in situ in the leaves of WT and OsANN4-R plants with or without ABA treatment. F Histochemical detection of $\mathrm{H}_{2} \mathrm{O}_{2}$ in situ in the roots of WT and OsANN4-R plants with or without ABA treatment. Scale bars for images E. and (f) are 
795 Fig. 3. OsANN4 promotes stomatal closure and alleviates $\mathrm{H}_{2} \mathrm{O}_{2}$-induced aerenchyma 796 formation with $\mathrm{ABA}$ treatment.

797 A Scanning electron microscopy images of three levels of stomatal apertures. B The percentage of 798 three levels of stomatal apertures in the leaves of OsANN4 RNAi and WT plants under normal and ABA treatment. Values are means $\pm \mathrm{SD}(23 \leq \mathrm{n} \leq 36) . * \mathrm{P}<0.05, * * \mathrm{P}<0.01$. C Cross sections at $3 \mathrm{~mm}$ and $5 \mathrm{~mm}$ from the tips of the adventitious roots in WT and OsANN4-R plants with $10 \mu \mathrm{M}$ ABA treatment for $12 \mathrm{~d}$. Sections were stained with toluidine blue. Scale bars are $20 \mu \mathrm{m}$. D The aerenchyma numbers in the adventitious roots of WT and OsANN4-R plants with ABA treatment. Values represent the mean \pm SD from three independent experiments. Statistical significance was determined by Student's t-test, $* \mathrm{P}<0.05, * * \mathrm{P}<0.01, * * * \mathrm{P}<0.001$

805

806

807

808

809

810

811

812

813

814

815

816

817

818

819

820 
Fig. 4. OsANN4 can interact with the protein kinase OsCDPK24.

826 A Yeast two-hybrid system was used to detect the interaction between OsANN4 and OsCDPK24. B A

827 GST pull-down assay was used to detect the interaction between OsANN4 and OsCDPK24. C Firefly

828 luciferase complementation imaging (LCI) assay detecting the interaction between OsANN4 and

829 OsCDPK24. The colored scale bar indicates the luminescence intensity.

830

831

832

833

834

835

836

837

838

839

840

841

842

843

844

845

846

847

848

849

850 
Fig. 5. OsANN4 is a $\mathrm{Ca}^{2+}$-binding protein that can be phosphorylated by OsCDPK24

856 A An in vitro kinase assay was performed with purified OsANN4-His and OsCDPK24-His using a

857 Phos-tag gel. Signals were detected by using a His-tag antibody. B Mass spectrometry analysis of 858 OsANN4 phosphorylation. C The fluorescence excitation of the purified OsANN4-His protein. D The

859 fluorescence excitation of the purified OsANN4(S13A)-His protein. E The emission spectra of the 860 purified OsANN4-His protein. F The emission spectra of the purified OsANN4(S13A)-His protein.

861

862

863

864

865

866

867

868

869

870

871

872

873

874

875

876

877

878

879

880 
885 Fig. 6. The interaction between OsANN4 and OsCDPK24 involved in response to 886 ABA signals

887 A Firefly luciferase (LUC) complementation imaging(LCI) assay detecting the interaction between 888 OsANN4 and OsCDPK24 with or without ABA treatment. The colored scale bar indicates the luminescence intensity. B Quantification of the relative cold luminescence signals displayed in the two group of A.. Values represent mean $\pm \mathrm{SD}$ and the single asterisk represent a significant difference determined by the Student's test, ${ }^{*} \mathrm{P}<0.05, \mathrm{n}>13$. C Firefly luciferase complementation imaging (LCI) assay detecting the interaction between OsANN4-(S13A) and OsCDPK24 with ABA treatment. The colored scale bar indicates the luminescence intensity. D Quantification of the relative cold luminescence signals displayed between OsANN4-(S13A) and OsCDPK24 with ABA treatment. 
Fig. 7. A proposed model for OsANN4 in response to ABA

916 The conformation of PYL can be changed after binding ABA, thus enhancing the stability of the

917 complexes of PYL and PP2C, resulting in reducing the inhibitory effect of PP2C on OsCDPK24.

918 OsCDPK24 interacts with OsANN4 and further phosphorylates OsANN4. OsANN4 enhances SOD

919 and CAT activities to scavenge $\mathrm{H}_{2} \mathrm{O}_{2}$. As a consequence, OsANN4 can further alleviate the formation

920 of aerenchyma. Under ABA treatment, the interaction between OsANN4 and OsCDPK24 is

921 strengthened, and OsANN4 may be more inclined to be phosphorylated by OsCDPK24.

922

923

924

925

926

927

928

929

930

931

932

933

934

935

936

937

938

939

940

941

942

943

944

945

946

947

948

949 
bioRxiv preprint doi: https://doi.org/10.1101/519199; this version posted January 13,2019 . The copyright holder for this preprint (which was not certified by peer review) is the author/funder. All rights reserved. No reuse allowed without permission.

950

951

952 


\section{$\mathbf{A}$}
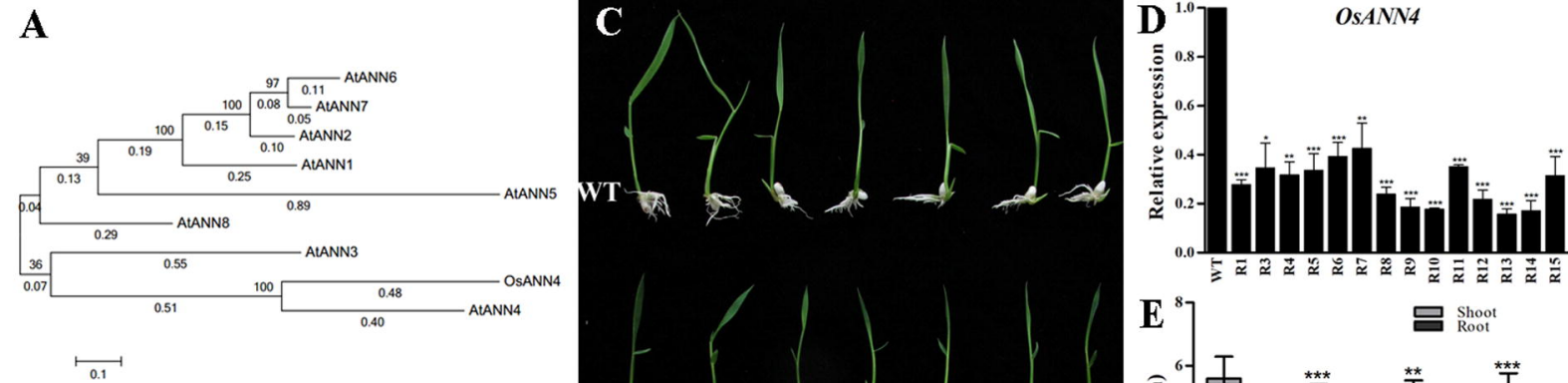

\section{B}

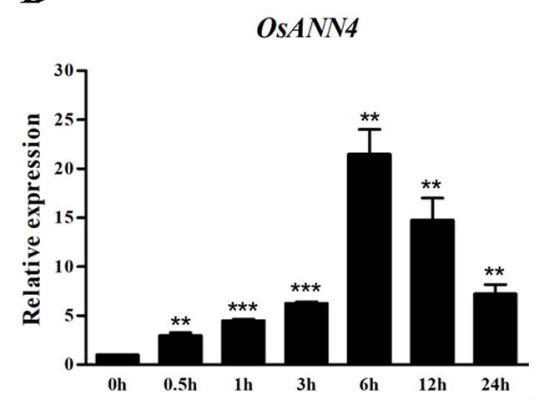

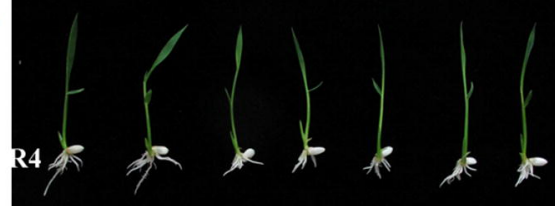

E

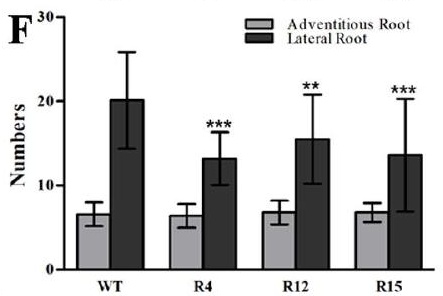

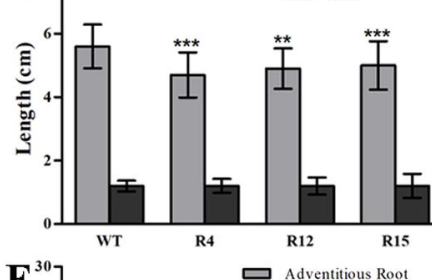




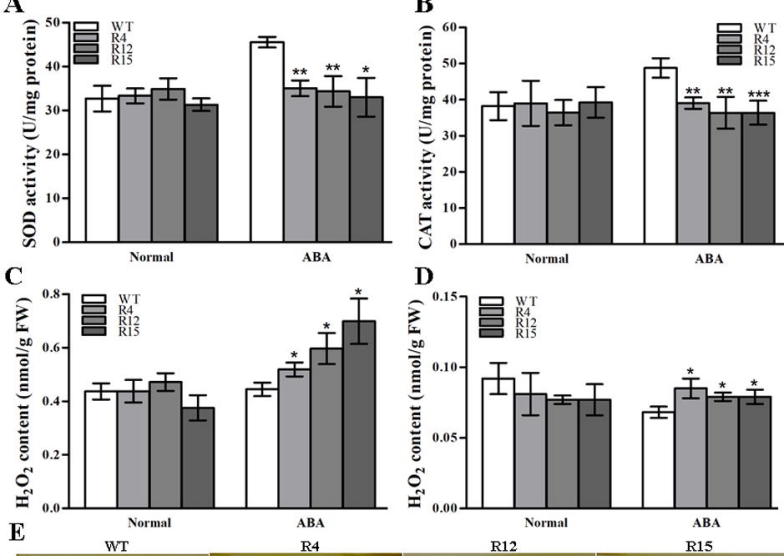

$\begin{array}{ll}\text { A } & \text { B }\end{array}$

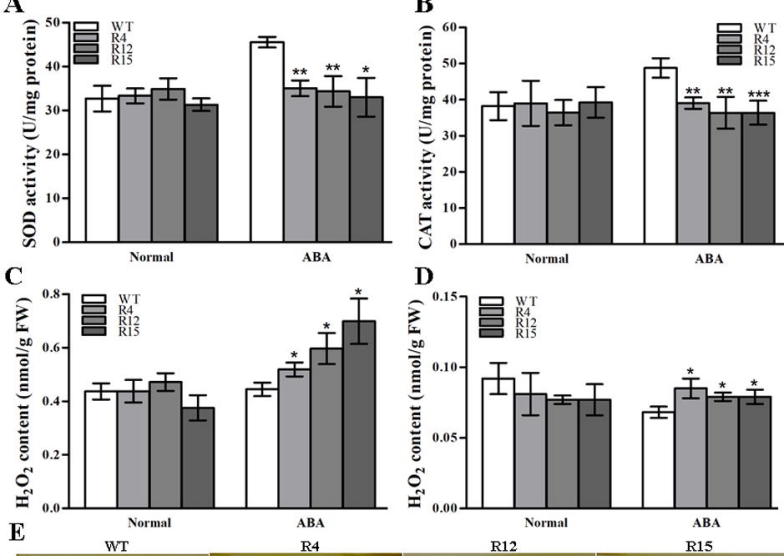

C

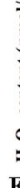

${ }^{0.8} 7$ 号 ${ }_{\mathrm{R} 12}^{\mathrm{RT}}$

兵

현 WT
D
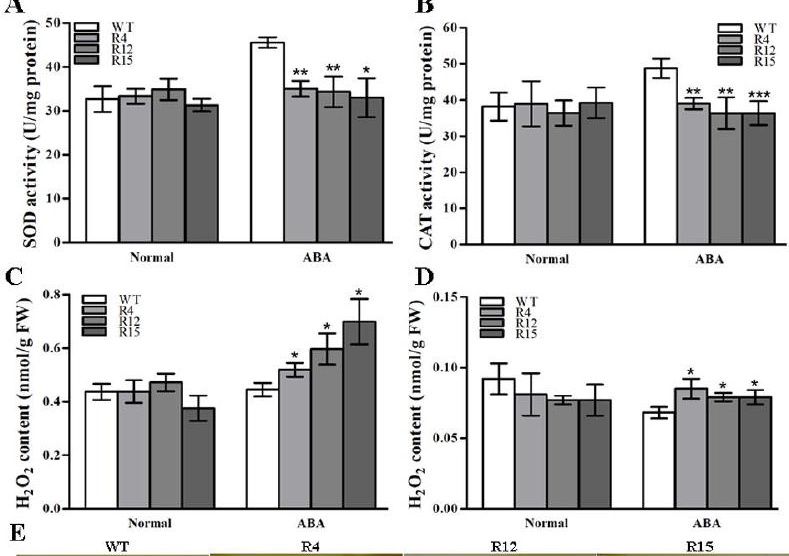
R4 4

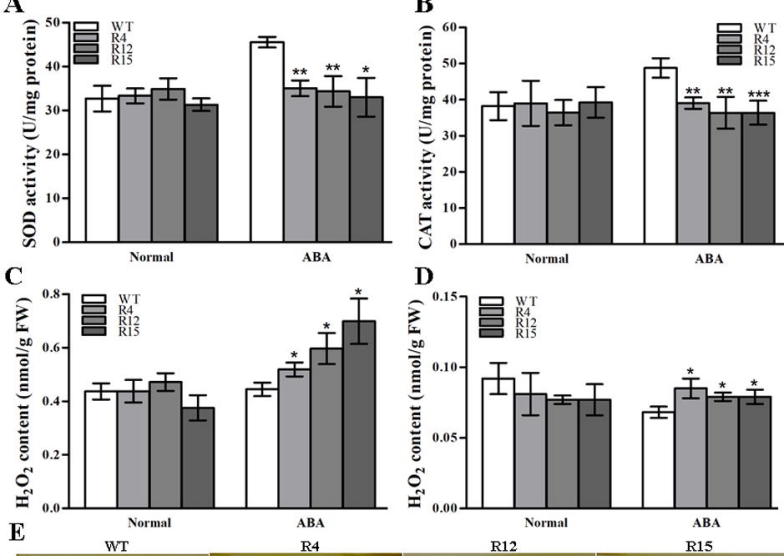
F
WT

R4
R12

R15
WT

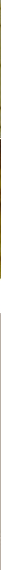

R12

Normal 
$\mathbf{A}$

Positive control

OsCDPK24-AD

+OsANN4-BD

Negative control

B \begin{tabular}{ccc}
-LWHA & -LW \\
\hline
\end{tabular}

\begin{tabular}{ccc}
-LWHA & -LW \\
\hline
\end{tabular}

C

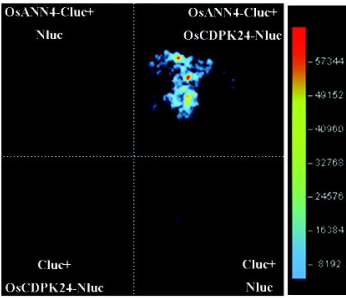

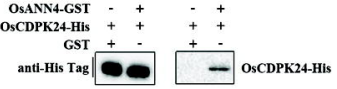

anti-GST Tag
OsANN4-GST

GST

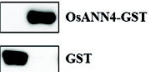

pull-down 


\section{A OsCDPK24-His}

OsANN4-His

His

$5 \mu \mathrm{M} \mathrm{Ca}^{2+}$

$500 \mu \mathrm{M} \mathrm{Ca}^{2+}$

$0.2 \mathrm{mM}$ ATP

$60 \mathrm{KD}$

+Phos Tag

40KD -
C
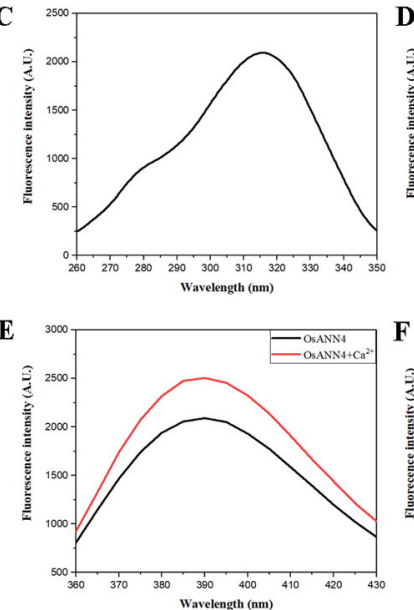
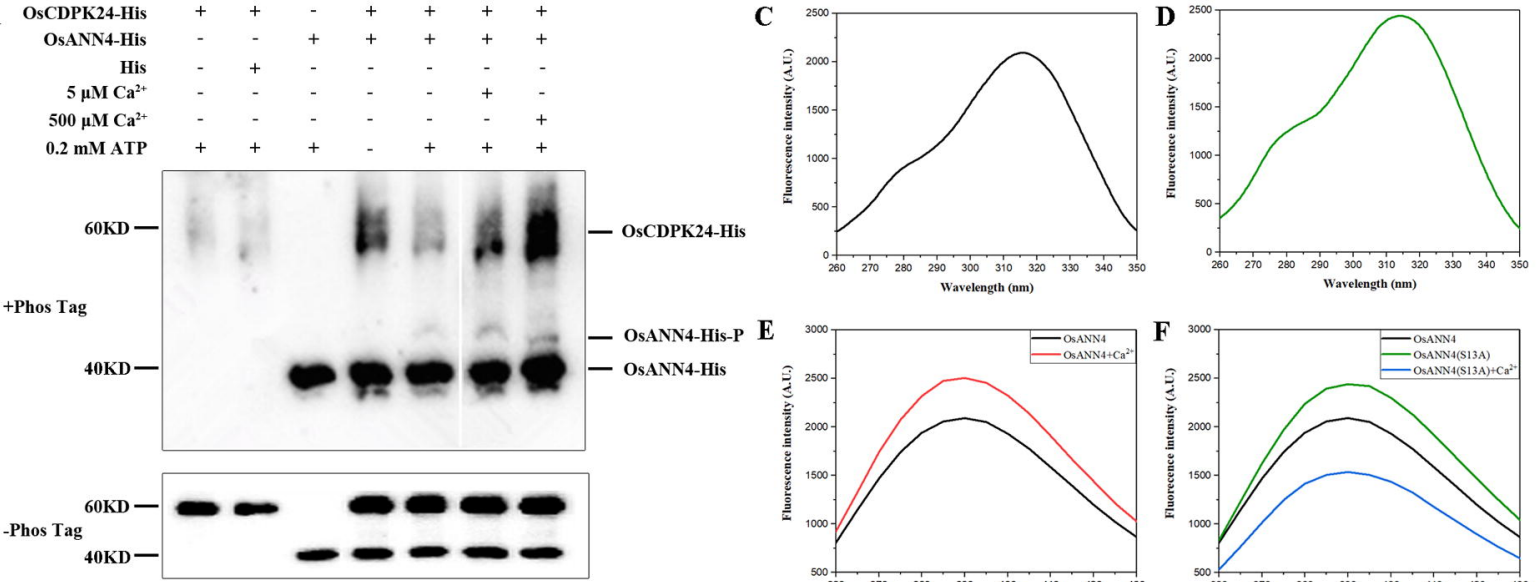

- OsANN4-His-P E

- OsANN4-His

B

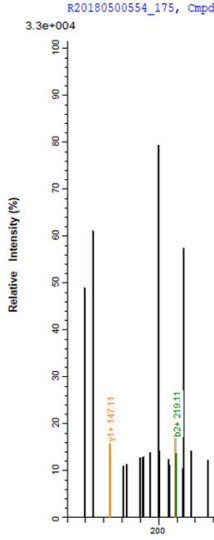

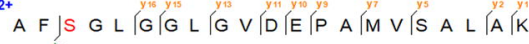

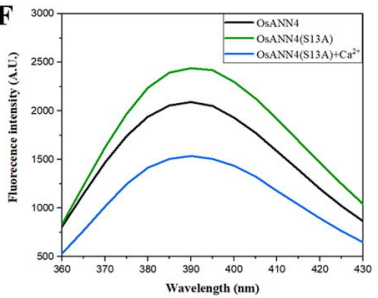

1 1

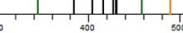

ะ่อ

100

100

1000

${ }_{1200}^{1}$

1,100

${ }_{1400}^{1}$ 


\section{$\mathbf{A}$}

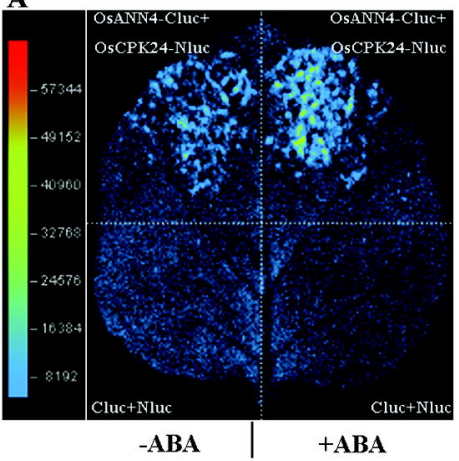

B

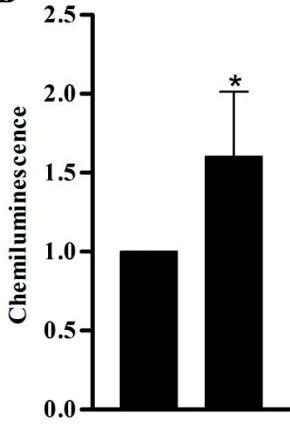

$-\mathbf{A B A}+\mathbf{A B A}$

D

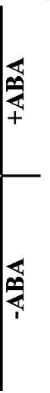

OsANN4(S13A)-Cluct OsCPK24-Nue
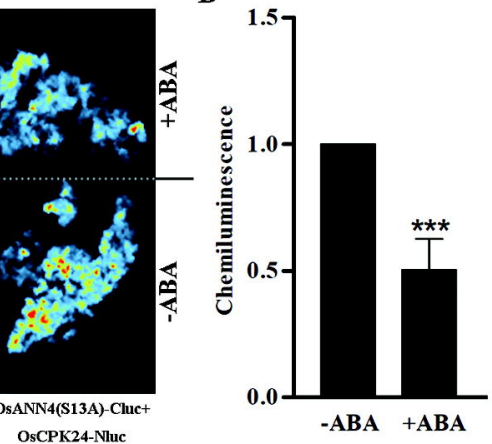

$-\mathbf{A B A}+\mathbf{A B A}$ 
